\title{
The nutritive value of groundnut protein
}

\author{
2.* The correlation between electrophoretic pattern \\ and nutritive value
}

\author{
BY R. DAWSON \\ Rowett Research Institute, Bucksburn, Aberdeen
}

(Received 30 October 1967-Accepted I March 1968)

\begin{abstract}
1. The effects of heat treatment on the proteins of the groundnut were studied by examining the electrophoretic patterns of the proteins extracted from samples of a laboratoryprepared groundnut flour after various heat treatments.

2. Marked changes in the electrophoretic pattern of the conarachin fraction were found and these changes correlated with changes in nutritive value.

3. Marked differences were found between the electrophoretic patterns of the conarachin fractions extracted from twenty commercial groundnut meals, and these differences could be correlated with nutritive value.

4. It is suggested that an examination of the electrophoretic pattern of the conarachin fraction could provide a useful and rapid means of determining the nutritive value of a groundnut meal.
\end{abstract}

From fresh groundnuts, or from groundnut meal prepared without the application of heat, more than $90 \%$ of the total nitrogen can be extracted as the two globulins, arachin and conarachin (Moorjani \& Bhatia, I 948; Woodman \& Evans I948; Woodham \& Dawson, I966) with $5-10 \%(\mathrm{w} / \mathrm{v}) \mathrm{NaCl}$ solution. Nutritionally, arachin is a poor protein in that it supports very little growth in mice (Sauberlich, Pearce \& Baumann, I948), in rats (Sure, r920; Baernstein, r937-8) or in chicks (Woodham \& Dawson, I968), whereas conarachin supports good growth in rats (Sure, I920; Macheboeuf \& Tayeau, 1942) and in chicks (Woodham \& Dawson, 1968).

Woodham \& Dawson (1966), studying the effects of controlled heat treatment on a laboratory-prepared defatted groundnut flour, found that as the severity of heat treatment increased the amount of protein extractable with $10 \%(\mathrm{w} / \mathrm{v}) \mathrm{NaCl}$ solution decreased. The conarachin fractions extracted from the heated samples of flour showed marked differences in amino acid composition (Dawson \& Woodham, I966), and as a possible explanation of the results it was suggested that the conarachin fraction consisted of several components which varied in their sensitivity to heat treatment.

Cama, Malik \& Nath (1958) found variations in the patterns obtained by moving boundary electrophoresis of the proteins extracted from groundnut meals prepared by different methods of processing and these variations correlated with variations in nutritive value of the meals. More recently an electrophoretic investigation of groundnut proteins was carried out by Tombs (1965), who demonstrated the existence of genetic variants of arachin which could be separated by electrophoresis on polyacrylamide gels. This technique showed that the proteins of groundnut could be resolved into considerably more than the four components reported by Cama et al.

* Paper no. I : Br. F. Nutr. (1968), 22, 589. 
(1958). It was decided therefore to carry out a study, using the technique of electrophoresis on polyacrylamide gel, of the saline-soluble proteins extracted from laboratoryprepared groundnut flour which had been subjected to various degrees of heat treatment, and also of the saline-soluble proteins extracted from commercial groundnut meals, in order to determine whether changes in electrophoretic pattern could be correlated with differences in nutritive value. Preliminary experiments showed that no significant changes in the electrophoretic pattern of the arachin fraction could be observed but significant differences were found in the conarachin fraction. This communication therefore describes the variation in electrophoretic pattern of the conarachin fractions and discusses the correlation between electrophoretic pattern and the nutritive value of the groundnut meals.

\section{EXPERIMENTAL}

Materials. Defatted groundnut flour was prepared as described by Woodham \& Dawson (1968). For dry-heat treatment samples of this flour were heated in an electrically heated forced-draught laboratory drying oven; the moist-heated samples were prepared by steam-heating in a laboratory autoclave. For accurate control of the temperature a thermistor probe was inserted into the layer of groundnut flour, the temperature being continuously recorded on an external meter.

The commercial groundnut meals were those collected and distributed to the various laboratories participating in the Agricultural Research Council collaborative trial of protein quality tests (Duckworth, Woodham $\&$ McDonald, 196r).

Extraction of conarachin. A $5 \mathrm{~g}$ portion of each meal was stirred with $50 \mathrm{ml}$ of I0\% $(\mathrm{w} / \mathrm{v}) \mathrm{NaCl}$ solution at room temperature for $2 \mathrm{~h}$. The suspension was then centrifuged and the supernatant liquid filtered through glass wool and treated with I0 $\mathrm{ml}$. of $10 \%(\mathrm{w} / \mathrm{v}) \mathrm{CaCl}_{2}$ solution to precipitate the arachin (Tombs, 1965$)$. The arachin was removed by centrifuging and the supernatant liquid was dialysed overnight against running tap water, causing precipitation of the conarachin. This was removed by centrifuging, washed with distilled water and dried in a vacuum desiccator over $\mathrm{CaCl}_{2}$.

For electrophoresis a $0.6 \%$ solution of each conarachin sample was prepared by dissolving $15 \mathrm{mg}$ of the conarachin in $2.5 \mathrm{ml}$ of the tris-citrate buffer used for preparing the polyacrylamide rods (see below) and containing $10 \%(\mathrm{w} / \mathrm{v})$ sucrose to increase the density of the solution. These solutions were prepared immediately before use.

Electrophoresis. The electrophoresis was carried out on rods of polyacrylamide gel in a Shandon Disc Electrophoresis Apparatus (Shandon Scientific Co. Ltd, London).

The gels contained tris-citrate buffer (0.076 M-tris-0.005 M-citric acid, $\mathrm{pH} 8.6)$ and the buffer tanks $\mathrm{NaOH}-\mathrm{NaBO}_{3}(0.30 \mathrm{M}$-boric acid-0.06 $\mathrm{M}$-sodium hydroxide, $\mathrm{pH} \mathrm{8 \cdot 48).}$

The $7.5 \%$ polyacrylamide gel rods were prepared by dissolving $\mathrm{r} \cdot 88 \mathrm{~g}$ Cyanogum 4I (BDH Chemicals Ltd) in $24 \mathrm{ml}$ tris-citrate buffer, $\mathrm{pH} 8 \cdot 6$. Dimethyl amino propionitrile (DMAP) $(0.075 \mathrm{ml})$ was added with gentle stirring and the solution filtered. 
Freshly prepared $7 \%(\mathrm{w} / \mathrm{v})$ ammonium persulphate solution $(\mathrm{r} \mathrm{ml})$ was then added with stirring and the solution quickly pipetted into the running tubes, care being taken to ensure that no air bubbles were trapped. The gel solution in the tubes was layered with distilled water, to prevent the inhibition of polymerization by atmospheric oxygen. The solution gelled within $10 \mathrm{~min}$. When ready for use the water layer was decanted from the tube and the surface of the gel washed with distilled water. The tubes were then placed in the electrophoresis apparatus and the space above the gel in each tube was filled with the tris-citrate buffer.

The samples of conarachin solutions were applied to the gel rods by layering the sample beneath the buffer in the tubes using a micro-pipette. For each analysis $25 \mu \mathrm{l}$ of the $0.6 \%$ solutions were used and electrophoresis was carried out for $\mathrm{I} h$, the current being equivalent to $4 \mathrm{~mA}$ per tube.

After electrophoresis the gel rods were removed from the running tubes and transferred immediately to tubes containing I $\%(\mathrm{w} / \mathrm{v})$ naphthalene black $\mathrm{I} 2 \mathrm{~B}$ in methanolacetic acid-water (500:200:500) for $\mathrm{r} h$. The stain was then decanted and the rods were washed with two changes of $7 \%(\mathrm{v} / \mathrm{v})$ acetic acid. Excess stain was then removed from the rods electrolytically.

\section{RESULTS}

In the following sections the interpretation of the electrophoretic patterns is based on the work of Tombs ( 1965 ).

Laboratory-prepared groundnut flour. The electrophoretic patterns of the conarachin fractions extracted from the samples of laboratory-prepared groundnut flour subjected to various heat treatments are shown in Pl. $1 a, b$. From Pl. $1 a$ it will be seen that there are no marked differences between the conarachin fraction from unheated flour and that from the flour heated at $125^{\circ}$ for $30 \mathrm{~min}$. After heating at $\mathrm{I} 25^{\circ}$ for $5 \mathrm{~h}$, however, there was an obvious decrease in the $\alpha$-conarachin component, and the $\beta$-conarachin components were intensified but more diffuse. After heating at $\mathrm{I}_{5} 0^{\circ}$ for $\mathrm{I} h$ the $\alpha$ conarachin component had almost completely disappeared, and the $\beta$-components were even more intense and diffuse than after heating at $125^{\circ}$ for $5 \mathrm{~h}$.

The effect of moist-heat treatment on the electrophoretic pattern of the conarachin fractions is shown in Pl. $\mathrm{I} b$. After moist-heat treatment of $108^{\circ}$ for $15 \mathrm{~min}$ the intensity of the $\alpha$-component and of the $\beta_{2}$-component increased and that of the remaining $\beta$-components decreased. Moist-heat treatment of $108^{\circ}$ for $45 \mathrm{~min}$ similarly increased the intensity of the $\alpha$-and the $\beta_{2}$-components and the number of $\beta$-component bands was reduced.

Commercial groundnut meals. The electrophoretic patterns of the conarachin fractions extracted from the commercial groundnut meals are shown in P1. 2. In this plate the polyacrylamide rods have been arranged in decreasing order of nutritive value of the meals as determined by the modified GPv technique of Duckworth et al. (196I). All the meals with a GPV greater than 48 showed a conarachin electrophoretic pattern similar to that obtained with the conarachin from the unheated laboratoryprepared groundnut flour but some differences could be observed, e.g. the $\alpha$-conarachin component was usually less intense than in the unheated flour, the $\beta_{2}$-component was 
more intense than in the unheated flour with a tendency for the intensity to increase with decrease in GPV, and the $\beta_{\mathbf{4}}$-component showed a decrease in intensity with decreasing GPv.

The electrophoretic patterns of the conarachin fractions from meals with GPvs less than 48 showed a number of differences when compared with the conarachin from the unheated flour. In some instances, e.g. GN I 5 and GN II, the patterns were quite different, with a very intense fast-running component. Other meals showed varying degrees of reduction of the $\alpha$-component, e.g. in $\mathrm{GN}_{4} 4$ and GN 2 the $\alpha$-component was missing completely and in GN 8 it was considerably reduced in intensity and much more diffuse. Also, there appeared to be variations in the $R_{F}$ value of the $\alpha$-component. Meals GN 19 and GN 20 were anomalous in that the electrophoretic patterns of their conarachin fractions were similar to those of meals having higher GPVs.

\section{DISCUSSION}

Woodham \& Dawson (1966) showed that the nutritive value of laboratory-prepared defatted groundnut flour decreased as the severity of heat treatment increased, and Dawson \& Woodham (1966) showed that the amino acid composition of the conarachin fraction extracted from samples of laboratory-prepared groundnut flour varied with the degree of heat treatment. The results obtained in this investigation demonstrate that these changes are paralleled by changes in the electrophoretic patterns of the conarachin fractions. Thus, increasing dry-heat treatment caused a progressive reduction of the $\alpha$-conarachin component while the $\beta$-conarachin components increased but became more diffuse and more difficult to resolve on electrophoresis. Moist-heat treatment, on the other hand, resulted in an apparent increase in the $\alpha$-conarachin with a decrease in the faster-running $\beta$-conarachin components.

The electrophoretic patterns of the conarachin fractions extracted from a variety of commercial groundnut meals with a wide variation in nutritive value were much more complicated and difficult to interpret than those obtained from the laboratoryprepared flour. This is not surprising, as it is likely that variations in electrophoretic patterns due to genetic and environmental differences will be superimposed upon those due to variations in processing conditions. Nevertheless, it is possible to make comparisons between the electrophoretic patterns of the laboratory-prepared samples and the commercial samples. Thus the electrophoretic pattern of the conarachin from GN 2 (GPV 32, Pl. 2) was very similar to that of the laboratory-prepared sample heated at $150^{\circ}$ for I h (GPV IO, Pl. I $a$ ) and the decrease in content of $\alpha$-conarachin in the commercial meals with GPvs less than 48 was reminiscent of the progressive reduction of $\alpha$-conarachin in the dry-heated laboratory-prepared samples.

One of the objects of this investigation was to determine whether there was any correlation between electrophoretic pattern and nutritive value. From the patterns in P1. 2, which are arranged in decreasing order of nutritive value, a definite trend can, in fact, be observed. Thus with the meals GN I2, GN $7, \mathrm{GN}_{5}$ and GN Io (GPvs 64, $5^{6}, 50$ and 48 respectively) the $\beta_{2}$-conarachin component increased and the $\beta_{4}$ - component decreased with decrease in GPV. As mentioned earlier, meals with GPVs below 48 showed a decrease in the $\alpha$-conarachin component and the $\beta_{2}$-component and this 
is well illustrated by the electrophoretic patterns of GN $x_{7}, \mathrm{GN}_{4} 4$ and GN 2 (GPvs 44,37 and 32 respectively Pl. 2). Thus in GN I 7 the $\alpha$-component was much reduced and the $\beta_{2}$-component was reduced and diffuse; in GN I4 the $\alpha$-component was reduced and the $\beta_{2}$-component was only just visible, and in GN 2 both the $\alpha$-component and the $\beta_{2}$-component completely disappeared.

Apart from a few exceptions which are discussed below, the remainder of the commercial meals can be graded in order of nutritive value with a reasonable degree of accuracy by means of the electrophoretic pattern of their conarachin fractions according to the scheme outlined below:

(I) If the electrophoretic pattern of the conarachin shows a well-defined and intense band of $\alpha$-conarachin and if bands corresponding to both $\beta_{2^{-}}$and $\beta_{4}$-conarachin are present the groundnut meal has a GPV of at least 48 .

(2) If the $\beta_{2}$-component is more intense than the $\beta_{4}$-component, i.e. if the ratio of the $\beta_{2}$-component to the $\beta_{4}$-component is $>\mathrm{I}$, the GPV of the meal is between 48 and 55 . This ratio decreases with increase in GPV, so that at a GPV of 55 the ratio would be close to $\mathrm{I}$.

(3) If the ratio of $\beta_{2}$-component to the $\beta_{4}$-component is $<\mathrm{I}$ the GPV of the meal is greater than 55 . Again, the lower that ratio, the higher the GPV.

(4) With meals having GPvs between 42 and 48 the $\alpha$-, $\beta_{2}$ - and $\beta_{4}$-components are still visible in the electrophoretic pattern but the intensity of these bands decreases with decrease in GPV and one or more of them becomes diffuse, e.g. in GN 3 and GN 8 the $\alpha$-conarachin component is less intense and more diffuse than in meals with higher GPvs. In GN 17 the $\beta_{2}$-component is less intense and diffuse. Also in this range of GPvs the components faster-running than the $\beta_{4}$-component tend to aggregate into fewer but more intense bands than are found in the patterns of meals with higher GPVs. This can be seen for both GN 8 and GN 17 .

(5) With meals having GPvs below 42 one or more or the $\alpha$-, $\beta_{2}$ - or $\beta_{4}$-components are usually absent from the electrophoretic pattern, e.g. in GN I5 and GN i $\alpha$-conarachin is completely missing. In GN $\mathrm{I}_{4}$ the $\beta_{2}$-component is barely visible and in $\mathrm{GN} z$ the $\alpha$ - and $\beta_{2}$-components are missing. Resolution of the remaining components is poor, particularly that of the faster-running ones.

The exceptions referred to earlier are as follows: GN I 8 was found to have a GPV of $5 \mathrm{I}$ but the electrophoretic pattern of its conarachin fraction showed a reduction in its $\alpha$-conarachin component and the over-all pattern was more like that of GN I 7 with a GPV of 44. GN I9 (GPV 36) showed a well-defined electrophoretic pattern for its conarachin and on this basis was more like $\mathrm{GN} \mathrm{I}_{3}$ (GPV 50). Similarly, GN 20 (GPV 36) showed a well-defined electrophoretic pattern similar to that of GN Io (GPV 48). In this connexion it is interesting that meals GN 19 and GN 20 were also found to be atypical by Barnes \& Woodham (1963), who investigated the correlation between nutritive value and nitrogen solubility. The solubility values suggested that both these meals should have higher nutritive values than had been suggested by the GPV technique.

Finally GN II (GPV 36 ) and GN I5 (GPV 40) had electrophoretic patterns very different from those of the other meals but in both there was no component corres- 
ponding to $\alpha$-conarachin, thus indicating low GPvs in keeping with the GPvs found by measurement.

Although the examination of the electrophoretic patterns of groundnut conarachins probably gives no more information regarding nutritive value, and may take a little longer to carry out than does the determination of salt-soluble nitrogen (Barnes \& Woodham, 1963 ) it does, however, provide information as to the effects of processing conditions on specific components of the groundnut proteins. Unfortunately, the changes in electrophoretic pattern are complex, involving more than one component, but nevertheless a reasonably accurate assessment of the nutritive value of the groundnut meal may be obtained from a study of the over-all electrophoretic pattern of the conarachin fraction. An attempt was made to make the technique quantitative by scanning the gel rods in a densitometer and comparing the intensities of various bands but this was not successful. This confirmed the view expressed by Tombs \& Akroyd (1967): 'For most purposes, evaluation of the destained gels by careful visual inspection together with photographic recording is sufficient. Densitometric curves can be obtained from either a photograph or the gel rod itself, but they do not give more information about band density and migration distance than a simple photograph. There may be $20-30$ bands in a $35 \mathrm{~mm}$ length of gel rod, some a fraction of a millimetre wide and separated by only a fraction of a millimetre, and a rooo-fold variation in intensity of light absorption. Several bands may be separated by regions of only slightly lower intensity staining; these are detectable on inspection of the gel, but may be missed completely in a densitometric scan. Accurate quantitative measurements are difficult because of the usual difficulties of varying uptakes of dye by different proteins and by small variations in the staining/destaining procedure, which would require many calibration gels of different concentrations of model proteins.'

It should be mentioned that the GPV technique by means of which the nutritive values of the meals were determined is a relatively imprecise method, individual values for a range of groundnut meals being subject to a standard error of \pm 6 units (Boyne, Carpenter \& Woodham, I96I). Further, a number of years has elapsed since the GPV determinations were made and although the meals were stored at $-10^{\circ}$ in sealed cans under nitrogen during this time it is possible nevertheless that changes could have occurred which might have affected the nutritive value of the meals. However, despite these factors and the possibility that genetic and environmental variations might also affect the electrophoretic patterns of the groundnut conarachin fractions, it appears that these patterns can provide a useful and rapid means of assessing the nutritive value.

I wish to thank Miss Alice Sargent and Mr A. D. McIntosh for their assistance during. the course of this investigation.

\section{REFERENCES}

Baernstein, H. D. (1937-8). F. biol. Chem, 122, 78I.

Barnes, M. McC. \& Woodham, A. A. (1963). F. Sci. Fd. Agric. 14, 109.

Boyne, A. W., Carpenter, K. J. \& Woodham, A. A. (I961). F. Sci. Fd. Agric. 12, 832.

Cama, H. R., Malik, D. A. \& Nath, R. (1958). F. Nutr. 66, 123. 
British Yournal of Nutrition, Vol. 22, No. 4

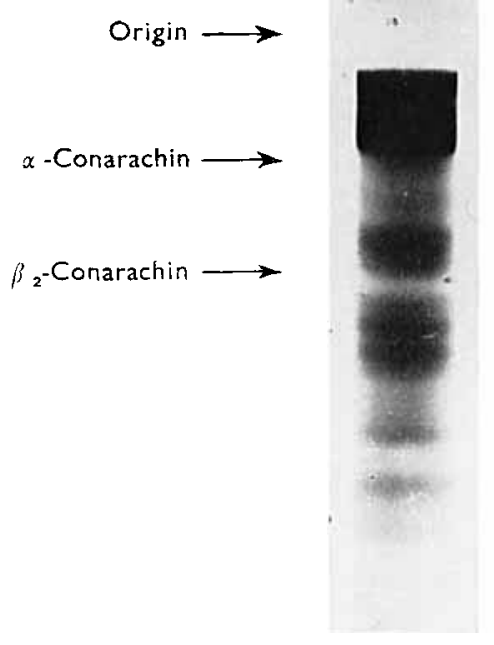

GPV

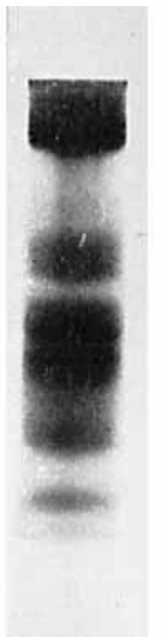

$125^{\circ} / 30 \mathrm{~min}$ 47

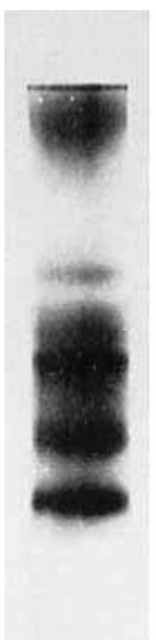

$125 \% 5 \mathrm{~h}$ 32

(a)

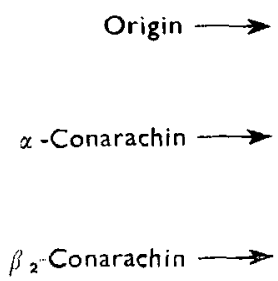

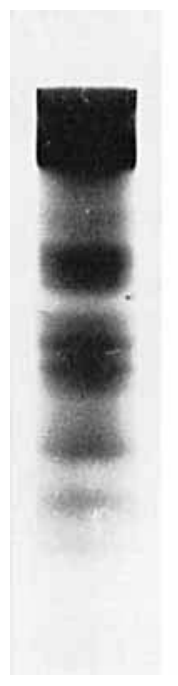

Unheated 48

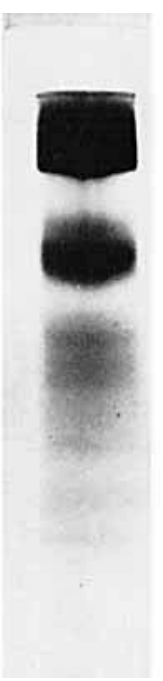

$108^{\circ} / 15 \mathrm{~min}$ 47
Plate I
$(+)$ $150^{\circ} / 1 \mathrm{~h}$ 10

$\theta$

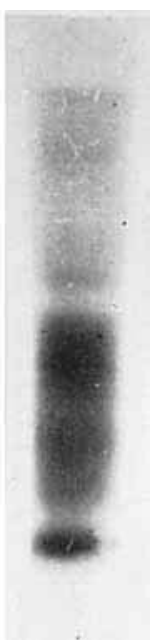

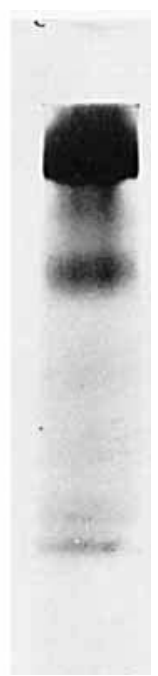

(1)

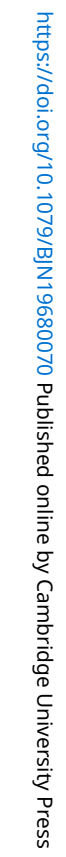

$\ominus$

$108^{\circ} / 45 \mathrm{~min}$

50 

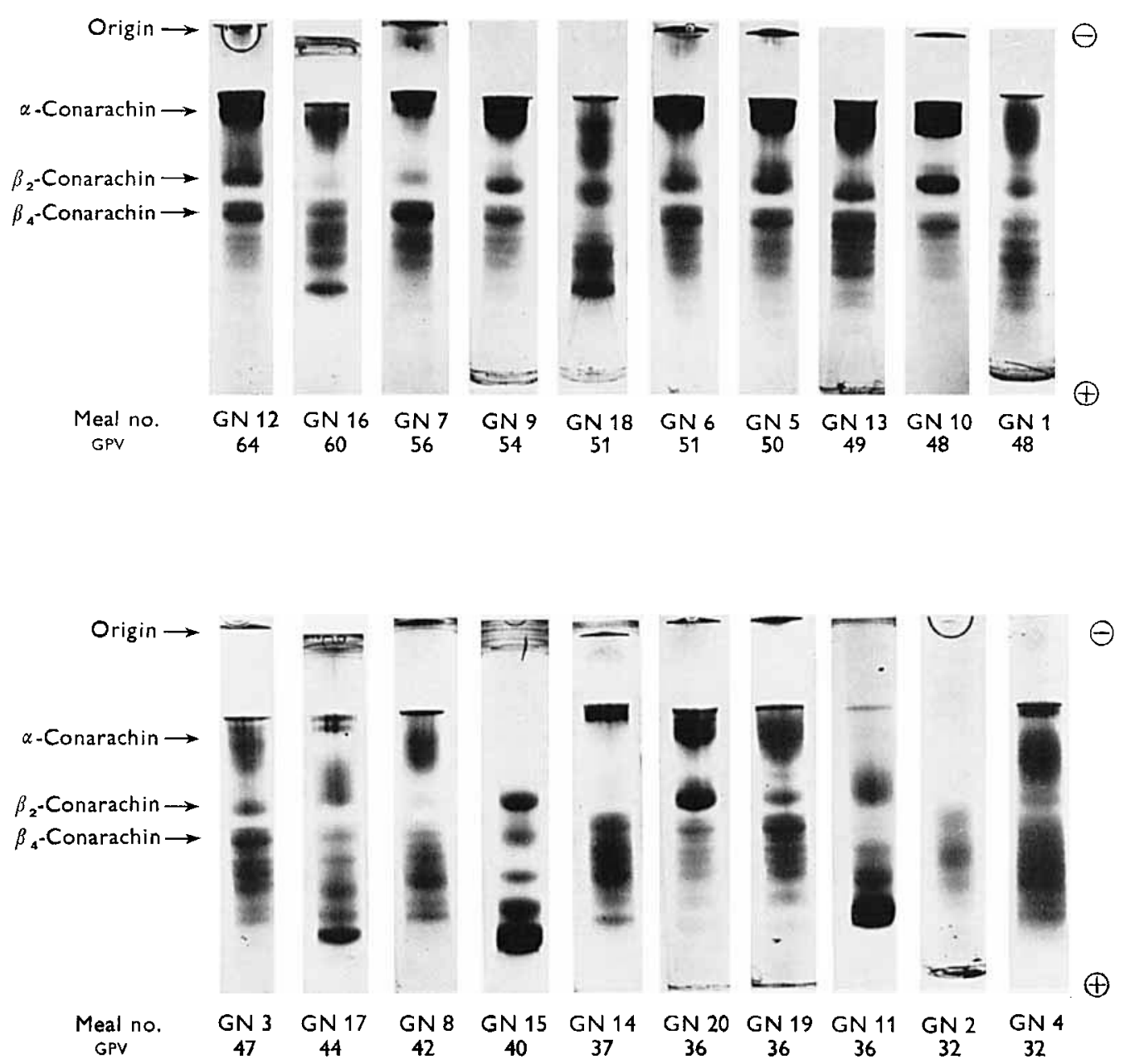

R. DAWSON 
Dawson, R. \& Woodham, A. A. (I966). Proc. Nutr. Soc. 25, ix.

Duckworth, J., Woodham, A. A. \& McDonald, I. (I961). F. Sci. Fd. Agric. 12, 407.

Macheboeuf, M. \& Tayeau, F. (1942). Bull. Soc. chim. biol. 24, 277.

Moorjani, M. N. \& Bhatia, D. S. (1948). F. scient. ind. Res. I3 B, I 13.

Sauberlich, H. E., Pearce, E. L. \& Baumann, C. A. (1948). J. biol. Chem. 175, 29.

Sure, B. (1920). F. biol. Chem. 43, 443.

Tombs, M. P. (1965). Biochem. F. 96, 119.

Tombs, M. P. \& Akroyd, P. (1967). Shandon Instrument Applications, no. 18. London: Shandon Scientific Co. Ltd.

Woodham, A. A. \& Dawson, R. (I966). Proc. Nutr. Soc. 25, viii.

Woodham, A. A. \& Dawson, R. (1968), Br. F. Nutr. 22, 589.

Woodman, H. E. \& Evans, R. E. (1948). F. agric. Sci., Camb. 38, 200.

\section{EXPLANATION OF PLATES}

Plate $x$

(a) The electrophoretic patterns of the conarachin fractions extracted from samples of a laboratoryprepared groundnut flour subjected to various dry-heat treatments.

(b) The electrophoretic patterns of the conarachin fractions extracted from samples of a laboratoryprepared groundnut flour subjected to different moist-heat treatments.

\section{Plate 2}

The electrophoretic patterns of the conarachin fractions extracted from a series of commercial groundnut meals differing in nutritive value. 